\title{
Formation Scenarios for Globular Clusters and Their Host Galaxies
}

\author{
Stephen E. Zepf \\ Dept. of Physics and Astronomy, Michigan State University, and \\ Department of Astronomy, Yale University, USA
}

\begin{abstract}
.
This review focuses on how galaxies and their globular cluster systems form. I first discuss the now fairly convincing evidence that some globular clusters form in galaxy starbursts/mergers. One way these observations are valuable is they place important constraints on the physics of the formation of globular clusters. Moreover, it is natural to associate the typically metal-rich clusters forming in mergers with the substantial metal-rich population of globulars around ellipticals, thereby implying an important role for galaxy mergers in the evolution of elliptical galaxies. I also highlight some new observational efforts aimed at constraining how and when elliptical galaxies and their globular cluster systems formed. These include systematic studies of the number of globular clusters around galaxies as a function of morphological type, studies of the kinematics of globular cluster populations in elliptical galaxies, and a variety of observational programs aimed at constraining the relative ages of globular clusters within galaxies as a function of cluster metallicity. The understanding of the formation of globular cluster systems and their host galaxies has grown dramatically in recent years, and the future looks equally promising.
\end{abstract}

\section{Introduction}

The study of the formation of globular cluster systems and their host galaxies has progressed greatly in recent years. Two of the main subjects of this meeting - the formation of objects with the properties of young globular clusters in nearby galaxy starbursts/mergers, and the presence of distinct globular cluster populations around elliptical galaxies - were controversial ideas outside of the mainstream at the last major globular cluster conference in Santa Cruz about a decade ago. The goal of this paper is to place these advances in the context of models of the formation of globular cluster systems and their host galaxies, and to highlight critical areas for future progress. To discuss the formation of globular cluster systems and their host galaxies is a tall order. To provide some focus within this broad subject, I will orient this paper around three questions 1) How do globular clusters form (and evolve)? 2) Why do elliptical galaxies have more globular clusters than spirals (and how many more do they really have)? 3) Why do many elliptical galaxy globular cluster systems have bimodal color distributions? 


\section{Globular Cluster Formation}

There are several reasons for starting the discussion of the formation of globular cluster systems and their host galaxies with the question of how globular clusters themselves form. Firstly, the physical conditions required for globular cluster formation must have been in place during galaxy formation in order to account for the presence of globular clusters around nearly all galaxies. If we can determine the physical conditions that enable globular clusters to form, it follows that these must have been present during the formation of their host galaxies. Secondly, there has been dramatic recent progress observing the process of globular cluster formation in nearby mergers and starbursts. These observations directly show us at least one set of physical conditions that leads to the formation of star clusters with the sizes and masses of globular clusters.

As evidenced in the conference poster and many talks at this meeting, HST imaging has revealed a wealth of compact, luminous young star clusters in starbursts and mergers. These star clusters are observed to have the sizes, luminosities, and colors predicted for globular clusters at young ages (e.g. Ashman $\&$ Zepf 1992). Subsequent spectroscopy has confirmed their stellar nature and ages, and in a few cases their masses inferred from the colors and luminosities (see also many papers in these proceedings). Spectroscopy has also shown that the metallicities of these clusters are very roughly solar, as expected from clusters that form out of enriched gas in spiral galaxy disks. Moreover, there are now examples of star clusters with masses and densities like those of Galactic globular clusters at essentially all ages. These include systems ranging from birth (e.g. NGC 4038/4039, Whitmore et al. 1999) through youth at several hundred Myr (e.g. NGC 7252, Miller et al. 1997) to middle age at several Gyr (e.g. NGC 1316, Goudfrooij et al. 2001) to old age (e.g. the Galaxy and M87, Kundu et al. 1999).

A natural interpretation of these data is that (metal-rich) globular clusters form in gas-rich mergers, and that the formation process of galaxies with a significant population of metal-rich globulars was similar to the nearby galaxy mergers we see today. Since most ellipticals have a significant metal-rich globular cluster population, this is essentially saying that mergers play a major role in the formation of ellipticals. Moreover, since roughly half of the globular clusters in the local universe are "metal-rich" $([\mathrm{Fe} / \mathrm{H}] \gtrsim-1.0)$, many globular clusters may have formed in a similar fashion to that observed in nearby mergers, placing important constraints on GC formation models.

These are powerful conclusions, so it is worth examining in detail how they come about, and what are some avenues for further tests and exploration of these ideas. Firstly, it is important to emphasize that the conclusion only applies directly to clusters that are fairly enriched, because we only observe the formation of such clusters in nearby starbursts and mergers. Whether the physics involved in forming globular clusters in gas-rich mergers can be extended to low metallicity regimes is uncertain. This question is something Keith Ashman and I are working on, with the main challenge being that metal-poor clusters tend to live in lower density regions, in which it is harder to maintain high pressures like those in dense starbursting regions. Therefore, it is possible that low metallicity $([\mathrm{Fe} / \mathrm{H}] \lesssim-1.0)$ clusters have a different formation mechanism than that observed for high metallicity $([\mathrm{Fe} / \mathrm{H}] \gtrsim-1.0)$ clusters. Because elliptical 
galaxies generally also have a significant population of low metallicity clusters, this also leaves open the possibility that other processes not associated with starbursts/mergers contribute to the building up of elliptical galaxies.

A second question is whether all clusters with compact sizes and inferred masses like those of Galactic globulars are genuine globular clusters regardless of age. One concern in this regard that has been effectively addressed is whether the young clusters generally lack low-mass stars so that they will effectively disappear before reaching old age. This arises in large part because the stellar initial mass function in starbursts is not well-known and also because not all of the handful of young clusters observed with high spectral resolution and signalto-noise have dynamical mass estimates consistent with standard stellar mass functions (Ho \& Filippenko 1996, Filippenko \& Ho 1996, Smith \& Gallagher 2001). However, this concern is soundly laid to rest by the observations of the NGC 1316 intermediate age globular cluster system by Goudfrooij et al. (2001). These authors show convincingly through imaging and spectroscopy that there are many bright, dense clusters in this galaxy with ages of about 3 Gyr. This confirms the existence of intermediate age clusters that had been suspected based on photometry of the cluster systems of several galaxies (e.g. Whitmore et al. 1997, Georgakakis, Forbes, \& Brodie 2001). The existence of 3 Gyr old star clusters is simply not possible if these objects only have high-mass stars. Thus the continuous age range observed for massive, dense star clusters, from very young to almost a Hubble time, provides strong evidence that some of the dense young clusters we observe today will survive to ripe old age.

The only significant observational difference between young and old dense cluster systems is their mass function. Specifically, young cluster systems appear to have a mass function that is a power-law with increasing numbers down to the limit of the observations of a few $10^{4} M_{\odot}$, while old systems have a mass function that is similar to that of the young systems for masses greater than 1$210^{5} M_{\odot}$, but below this "turnover" mass the number of objects in old systems follows a very much shallower slope, so that it has many fewer low-mass objects. This difference in mass function has now been established for a number of both young and old systems (e.g. Fall \& Zhang 1999, Zepf et al. 1999, Carlson et al. 1998, Miller et al. 1997). The question is whether this difference reflects a fundamental physical difference between younger and older globular cluster systems, or whether this is a result of the dynamical evolution of cluster systems.

Dynamical evolution of globular clusters is a consequence of basic physics. It has also long been realized that dynamical effects will tend to destroy lower mass clusters preferentially (e.g. Fall \& Rees 1977, Spitzer 1987). Therefore, there is little question that in a qualitative sense, basic gravitational physics will tend to turn an initially power-law cluster mass function like that seen in young systems into something resembling the log-normal shape typical of old globular cluster systems. However, whether this actually works quantitatively remains a critical unanswered question. On the theoretical side, there has been much recent work on the subject (see talks at this meeting by Fall and Vesperini), but there is not yet a full consensus on whether dynamical evolution can produce a luminosity function as uniform as observed between galaxies and within galaxies from an initial mass function that is a power-law rising steeply to low masses. Observationally, perhaps the most critical question is whether intermediate cases 
can be found between the power-laws with no turnover down to several $10^{4} M_{\odot}$ in young systems and the log-normal distributions with a "turnover" around 1$210^{5} M_{\odot}$ in old systems. As discussed in Zepf et al. (1999) it is not necessarily surprising that no turnover has been found in current studies of young systems, and it will be hard to push these to lower masses because of contamination with individual stars (cf. Whitmore et al. 1999). The best bet to make this test seems to be to push observations of intermediate age systems of several Gyr old to very faint levels.

The fact that globular cluster formation is directly observed is also a great advance for the study of how globular clusters form. Prior to these observations, there were few direct constraints on the physical conditions that lead to globular cluster formation. The observations of globular cluster formation in nearby starbursts and mergers have changed this situation dramatically. One attempt to take advantage of these new observations to inform models of globular cluster formation is a paper Keith Ashman and I have recently written (Ashman \& Zepf 2001). A key point of our paper is that the high pressure observed in starbursts implies that any bound clouds that form in the ISM of the starburst will be much more compact than typical Galactic molecular clouds, and that for typical starburst pressures, clouds with globular cluster-like masses will have globular cluster-like radii. We also show that the recent observation of Zepf et al. (1999) that the young star clusters have a weak or absent mass-radius relation places strong constraints on formation models, since nearly all models start from clouds with a mass-radius relation. Specifically, we find that if the star formation efficiency in the progenitor cloud scales with the binding energy of the cloud, star clusters without a mass-radius relation may be formed from clouds with the virial mass-radius scaling observed for Galactic molecular clouds.

\section{Why do elliptical galaxies have more GCs than spirals?}

The origin and implications of the greater specific frequency (number normalized by galaxy luminosity or mass) of globular clusters around elliptical galaxies compared to spiral galaxies is a long-standing question. This difference was first used to argue that ellipticals are not formed by the mergers of spirals (e.g. van den Bergh 1990, Harris 1991), since the simple combination of spirals would result in ellipticals with the same normalized number of globular clusters as spirals. Schweizer (1987) and Ashman \& Zepf (1992) argued that globular clusters could form in gas-rich mergers, thereby resulting in more globular clusters around ellipticals relative to spirals if ellipticals form by merging spirals. Moreover, Ashman \& Zepf (1992) predicted that this would lead to bimodal metallicity distributions for globular cluster systems around elliptical galaxies. Specifically, elliptical galaxies formed by mergers would have a metal-poor population from the halos of the progenitor spiral galaxies, and a metal-rich population formed during the merger from the enriched gas in the disks of the spiral galaxies.

However, before venturing too far into the discussion of why elliptical galaxies have more globulars than spiral galaxies, it is useful to consider the actual observational constraints on the number of globular clusters around different galaxies. In Figure 1, I plot an updated version of the number of globular clusters normalized by the stellar mass of the galaxy against galaxy mass (cf. 


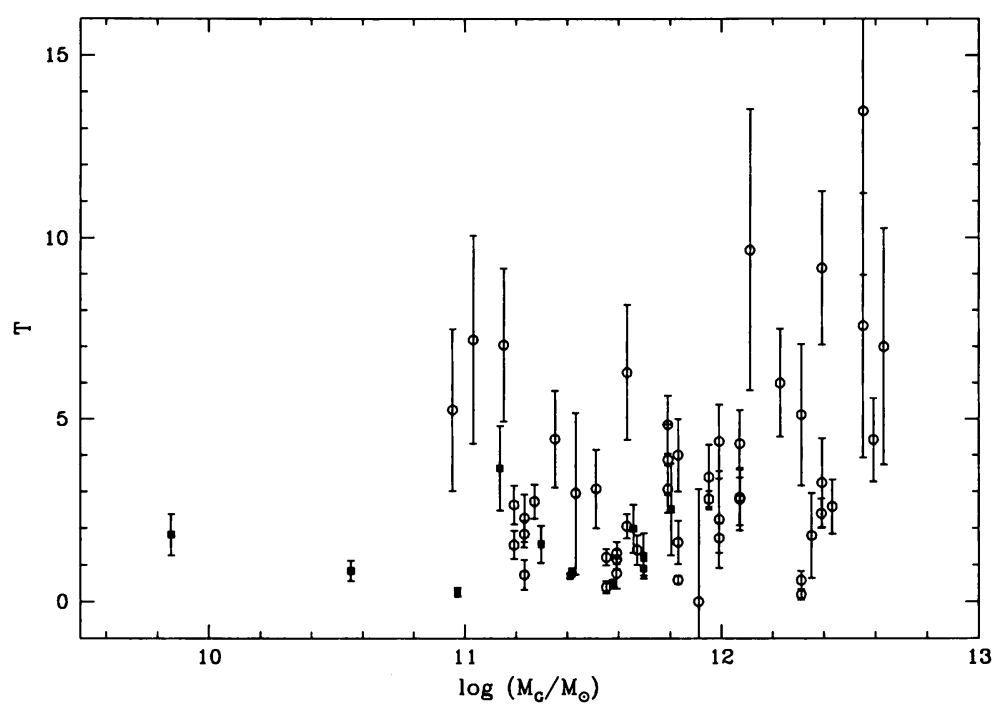

Figure 1. The mass-normalized specific frequency $T$ plotted against estimated galaxy mass. $T$ is defined as $T \equiv N_{G C} /\left(M_{G} / 10^{9} M_{\odot}\right)$. Early-type galaxies are plotted as open circles and spiral galaxies as filled squares. The error bars are only estimates of the statistical uncertainties and do not account for a number of possible systematic concerns. Most of the data are from Ashman \& Zepf (1998) where references are listed, with several updates from the literature that have generally decreased $T$ for some of the massive ellipticals.

Ashman \& Zepf 1998). The overall trend that elliptical galaxies, represented by open symbols, appear to lie above spiral galaxies, represented by closed symbols, seems clear from this figure. Thus, there is little question that per unit stellar mass, elliptical galaxies have more globular clusters than spiral galaxies. However, there is also tremendous scatter in this diagram.

What limits the comparison of ellipticals and spirals in this diagram? Firstly, there are very few well-studied spirals. The Milky Way and M31 dominate the comparison, along with M104, if it is counted as a spiral despite its very large bulge-to-disk ratio. This problem is being addressed in Katherine Rhode's PhD thesis, which includes high quality WIYN data for nine spiral galaxies. However, for now, the elliptical to spiral comparison suffers from having only a handful of well-studied spirals. A second major problem is the large uncertainties in the elliptical galaxy measurements of the specific frequency of globulars. This can be seen by the sizeable error bars in Figure 1. Moreover, these probably underestimate the true errors because they typically only account for statistical uncertainties and not systematic concerns such as the extrapolations to large radii often necessary to estimate the total number of globulars. Mosaic Camera data for the well-studied Virgo elliptical NGC 4472 suggest that its specific frequency is about $30 \%$ less than previously assumed (Rhode \& Zepf 2001). Similar results may now be coming out for M87 (Forte at this meeting). The obvious way to address this problem is with a systematic multi-color survey of early-type galaxies with the new generation of CCD Mosaic imagers. 
Despite the uncertainties, there are probably still some trends that show up in the data presented in Figure 1. One of these is that the specific frequency of globular clusters appears to increase slightly with increasing elliptical galaxy luminosity/mass (e.g. Djorgovski \& Santiago 1992; Zepf, Geisler, \& Ashman 1994, Kissler-Patig 1997). There are a number of plausible explanations for this effect. One possibility is dynamical evolution, because more luminous ellipticals have shallower density gradients, and thus destroy a smaller fraction of their clusters (e.g. Murali \& Weinberg 1997). Therefore, dynamical evolution will naturally produce at least some of the observed trend of $T$ with elliptical galaxy mass/luminosity. Another possibility is based on evidence that more massive ellipticals tend to have more gas mass. Therefore, the systematic trend of $T$ for massive ellipticals might be accounted for by adopting a constant number of globular clusters per baryon mass, rather than per stellar mass (e.g. Blakeslee, Tonry, \& Metzger 1997; McLaughlin 1999). Although the hope that the globular cluster frequency per "baryon mass" might be constant within a galaxy has also been ruled out recently in the best studied case of NGC 4472 (Rhode \& Zepf 2001), there is a good correlation for the most massive ellipticals between high specific frequency and large gas mass.

However, the most striking result of Figure 1, the systematic difference between the mass-normalized frequency of globular clusters around ellipticals relative to spirals, is hard to account for with either of these effects. The appeal to the hot gas as "missing stars" only works for massive ellipticals, as typical ellipticals around $L_{\star}$ do not have much mass in hot gas and may have a significant contribution to their X-ray luminosity from stellar sources (e.g. Sarazin, Irwin, \& Bregman 2000). Therefore, the difference in the mass-normalized globular cluster frequency $(T)$ between ellipticals and spirals of the same stellar mass is not solved by including mass in hot gas. Dynamical evolution is more physically plausible, since the disks and compact bulges of spiral galaxies can accelerate the destruction of clusters (e.g. Gnedin \& Ostriker 1997, Vesperini 1998), potentially resulting in fewer clusters around spirals compared to ellipticals. However, if the dynamical effects are strong enough to account for differences in specific frequency as large as those observed between ellipticals and spirals, they tend to produce significant differences between the mass functions of the different systems (e.g. Vesperini 2001). The strong similarity of the globular cluster luminosity functions of ellipticals and spirals (e.g. Whitmore 1997) therefore argues against dynamical evolution as the primary driver of the $T$ difference between spirals and ellipticals. Finally, it is possible to normalize the globular cluster numbers to bulge or halo luminosity (e.g. Harris 1991). In this case, the specific frequency of spiral bulges and/or halos can be similar to that of ellipticals (e.g. Forbes 2001), depending in large part on how the difficult task of determining bulge and/or halo luminosities is done for late-type galaxies. However, this approach does not address the critical question why disks like those of the Milky Way and M31 are unfavorable for globular cluster formation while the events that created the main bodies of elliptical galaxies were efficient at globular cluster formation, and what this means for how these galaxies formed.

The most appealing answer would seem to take a lesson from observations that quiescently star-forming disks are unfavorable for globular cluster formation, but actively starbursting and merging systems form globular clusters efficiently. Thus, if elliptical galaxies are those objects that had major mergers 
in their past, while spirals avoided such catastrophic events, the difference in their specific frequencies might be accounted for simply through the fact elliptical galaxies formed more of their stars in a mode favorable for globular cluster formation (Ashman \& Zepf 1992, Zepf \& Ashman 1993). Of course, such a proposal needs to be tested and compared to alternative pictures. Several efforts along these lines are discussed in the next section.

\section{Why are the globular cluster systems of elliptical galaxies bi- modal?}

Ashman \& Zepf (1992) suggested that elliptical galaxies formed by the mergers of disk galaxies would have two populations of globular clusters - a metal-poor population from the halos of the progenitor spiral galaxies, and a metal-rich population formed during the merger from the enriched gas in the disks of the spiral galaxies. This prediction is much different than the expectation of classical monolithic collapse models for elliptical galaxy formation in which a single metallicity peak is expected (e.g. Arimoto \& Yoshii 1987). Therefore, when bimodality was first discovered in the color distribution of elliptical galaxy globular cluster systems (Zepf \& Ashman 1993, Ostrov, Geisler, \& Forte 1993), it was a striking success for a prediction of a simple merger model.

Although the prediction of the simple merger model leads to bimodality, it is natural to ask what happens when one considers a more complex merger history involving several different major mergers over time, along with many lesser accretion events. While a complete calculation along these lines would require a detailed understanding of a number of challenging subjects such as feedback and chemical evolution, some progress can be made by treating the Milky Way as a typical disk galaxy, and considering what we know about its metallicity as a function of time. The critical aspect of the Milky Way in this regard is that the disk was enriched to near its current metallicity very early in its history ( $\mathrm{Ng} \&$ Bertelli 1998; Carraro, $\mathrm{Ng}$, Portinari \& 1998). If this is typical of disk galaxies, then it is natural that most of the globulars formed in mergers will have fairly high metallicity. It also follows from this argument that mergers of enriched, gas-rich disks will form elliptical galaxies with mostly metal-rich stars. This is consistent with observations that elliptical galaxies appear to have a G-dwarf problem (i.e. an absence of low metallicity stars compared to closed box models) similar to that in spirals (e.g. Harris \& Harris 2000, Marleau et al. 2000, Worthey, Dorman, \& Jones 1996).

Thus it seems plausible that even complex merger histories will produce globular cluster systems of many elliptical galaxies with generally bimodal color distributions. This arises because disks like that of the Milky Way were enriched to near solar metallicity very early, and thus mergers of these disks will produce clusters that are predominantly metal-rich, while the halos of the progenitor spirals have clusters that are predominantly metal-poor. Of course, globular clusters formed out of enriched gas in early disks will be unlikely to all have exactly the same metallicity. There will likely be radial gradients in the progenitor disks, and there will probably be a dependence of the metallicity of the disk on the galaxy luminosity/mass. In a merger-induced starburst, there will likely be additional enrichment, the amount of which will probably be tied to the depth of 
the potential well. Moreover, a few gas-rich mergers will have happened recently enough that the metal-rich clusters will be too young to be clearly redder than the old metal-poor clusters. However, observing these fine distinctions in typical color distributions will be very difficult because of photometric errors and uncertainties in the conversion of broad-band colors to metallicity. The result is that the roughly solar metallicity of the Milky Way disk over nearly all of the Hubble time suggests that most of the gas-rich merger events that build up elliptical galaxies and possibly other large bulge systems will produce globular clusters with metallicities somewhere near solar, and these may often result in bimodal color distributions for globular cluster systems.

Another natural question is the origin of the metal-poor cluster populations around early-type galaxies. In the simplest merger picture, the metal-poor clusters come from the halos of the progenitor spirals. However, it has long been recognized that this model appears to be inadequate to account for the full range of properties of the metal-poor cluster populations around ellipticals, if the halo populations of the Milky Way and M31 are typical of such progenitor spirals (e.g. Zepf, Ashman, \& Geisler 1995, Forbes et al. 1997, Kissler-Patig et al. 1998, Côté, Marzke, \& West 1998, Ashman \& Zepf 1998). In particular, a few ellipticals appear to be "missing" a significant metal-poor population (e.g. Woodworth \& Harris 2000, Zepf et al. 1995), while many very massive ellipticals appear to have a high specific frequency of metal-poor clusters. One of the weaknesses in all of these studies are the large and potentially systematic uncertainties in specific frequencies discussed in the previous section. For example, NGC 4472 has often been taken as a fiducial example of a massive elliptical with a high specific frequency, but better data from Mosaic CCD imaging suggests that the specific frequency of this galaxy is lower than previous estimates (Rhode \& Zepf 2001). Moreover, all of these analyses rely on the assumption that the Milky Way and M31 halos are typical of spiral galaxies. However, even with these serious systematic concerns, there are almost certainly some very massive ellipticals with higher mass-normalized specific frequencies for metal-poor clusters than can easily be achieved by combining typical spiral galaxies.

There are several possibilities for accounting for the large number of metalpoor globulars around some massive ellipticals. One is to consider that even the halos of the Milky Way and M31 appear to have been at least partially the result of accretion and merging over time. These processes would also be expected to occur both in the halos of the progenitor spirals and in the elliptical galaxy after it formed from major mergers. If for some reason, these elliptical galaxies accreted more low-mass galaxies, or the accreted galaxies had a higher specific frequency of metal-poor globulars, then it would be possible to make an elliptical with a larger mass-normalized specific frequency of metal-poor globulars. This process was modeled in detail by Côté et al. (1998), who found they could match the estimate of the metal-poor globular cluster population of NGC 4472 at that time, with a steep galaxy luminosity function $(\alpha \simeq-1.8)$ and a fairly high specific frequency of metal-poor globulars for dwarf galaxies. An alternative is to revisit the idea that the gas mass around the most massive ellipticals was available to form metal-poor globulars, but not stars (e.g. Blakeslee et al. 1997, Harris, Harris, \& McLaughlin 1998; McLaughlin 1999) These two ideas are not necessarily separate, since they both can be more or less accommodated in a model with a population of dwarf galaxies around these massive ellipticals that 
produces some globulars and not many stars in a burst, and then disperses, and is unable to cool again.

Perhaps the most interesting question is what observations can be made to shed further light on how elliptical galaxies and their globular cluster systems formed. Here I will highlight two such possibilities. One area that can shed light on the physical mechanisms involved in the formation of elliptical galaxies and their cluster systems is the study of the kinematics of the globular cluster systems. Because this is the subject of other recent reviews (e.g. Bridges 2001, Zepf 2001), I will only highlight one aspect of this work. A key question that the kinematics can address is the angular momentum of globular cluster populations. If galaxies generally form by dissipational collapse, they will spin-up as the result of the collapse and conservation of angular momentum (e.g. Fall \& Efstathiou 1980). In contrast, mergers provide a way to transport angular momentum outwards (e.g. Barnes \& Hernquist 1992). Therefore, it is potentially very interesting that of the three elliptical galaxies for which fairly detailed kinematics of the globular cluster systems exist (NGC 5128, Hui et al. 1995; M87, Cohen 2000, Côté et al. 2001; and NGC 4472, Zepf et al. 2000), the rotation is significantly smaller than the dispersion for two of these (M87 and NGC 4472). In both cases, rotation appears to become more important at larger radii and/or for the less centrally concentrated metal-poor cluster populations, also suggesting angular momentum transport.

Another critical observation is the relative ages of the metal-rich and metalpoor clusters. In general, merger models predict that the typical ages of the metal-rich clusters will be younger than the typical ages of the metal-poor clusters. Conversely, models that start with a monolithic collapse at the center, and accrete the metal-poor population afterwards tend to predict older ages for the metal-rich clusters compared to the metal-poor clusters. The challenge is that determining ages, even relatively, is difficult. Even for the Galaxy and M31 the situation is not completely clear, although there is tentative evidence that at least some of the metal-rich globulars in the Milky Way (Rosenberg et al. 1999) and M31 (Barmby, Huchra, \& Brodie 2001) are somewhat younger than the typical metal-poor globulars in these galaxies. Unsurprisingly, the situation is more difficult for globulars outside of the Local Group. Here I will review several of the techniques being applied, although they are far from definitive as yet. One common approach is to use the Balmer lines as age indicators. However, when applied to 47 Tuc and other metal-rich Galactic globulars, this gives ages of 20 Gyr (Gibson et al. 1999, Vazdekis et al. 2001), so there are clearly some unresolved systematic questions (cf. Cohen, Blakeslee, \& Ryzhov 1998, Beasley et al. 2000). In general the studies above find that the ages may be similar for cluster populations of different metallicities and that no cluster population is very young.

Another approach to determining the age differences between metal-rich and metal-poor populations is to compare their luminosity functions. Given the assumption the underlying mass functions are the same, the difference in the luminosity function can be turned into a difference in mass-to-light ratio, which can be combined with the observed color difference to estimate the relative age and metallicity of each population. When applied to M87, this technique appears to suggest the metal-rich population is somewhat younger than the metal-poor 
population (Kundu et al. 1999), and when applied to NGC 4472, the data tend to suggest fairly equal ages (e.g. Puzia et al. 1999). As discussed in these papers, the error bars on these techniques are significant, and the results are very dependent on the stellar population models applied and the assumption of equivalent mass functions for different metallicity populations.

An approach along somewhat similar lines is to use a wide enough spread of broad-band colors so that the age-metallicity degeneracy is broken to some extent. Puzia et al. (2001) and Kissler-Patig et al. (2001) have attempted this using VIK colors with some success. An independent approach is to use far-UV colors as a probe of the color of the horizontal branch, which also has an age dependence. Two HST studies along these lines (PIs Zepf and O'Connell) are being carried out now, and the results should be forthcoming next year. Overall, there are suggestions of modestly younger, metal-rich populations in elliptical galaxies, but it is still early days for this work.

Acknowledgments. This work is due in large part to my many excellent collaborators. I especially acknowledge my long-time collaboration with Keith Ashman. Some of the work presented here has been supported by NASA LongTerm Space Astrophysics grant NAG5-9651 and NASA Astrophysics Theory Program grant NAG5-9168, and my travel to this meeting was partially supported by an AAS travel grant.

\section{References}

Arimoto, N., \& Yoshii, Y. 1987, A\&A, 173, 23

Ashman, K.M., \& Zepf, S.E. 1992, ApJ, 384, 50

Ashman, K.M., \& Zepf, S.E. 1998, Globular Cluster Systems (Cambridge: Cambridge University Press)

Ashman, K.M., \& Zepf, S.E. 2001, AJ, in press (astro-ph/0107146)

Barmby, P., Huchra, J.P., \& Brodie, J.P. 2001, AJ, 121, 1482

Barnes, J. E., \& Hernquist, L. 1992, ARA\&A, 30, 705

Beasley, M.A., et al. 2000, MNRAS, 318, 1249

Blakeslee, J. P., Tonry, J. L., \& Metzger, M. R. 1997, AJ, 114, 482

Bridges, T.J. 2001, these proceedings

Carlson, M.N. et al. 1998, AJ, 115, 1778

Carraro, G., Ng, Y.K., \& Portinari, L. 1998, MNRAS, 296, 1045

Cohen, J.G. 2000, AJ, 119, 162

Cohen, J.G., Blakeslee, J. P., \& Ryzhov, A. 1998, ApJ, 496, 808

Côté, P., Marzke, R.O., \& West, M.J. 1998, ApJ, 501, 554

Côté, P., et al. 2001, ApJ, in press

Djorgovski, S.G., \& Santiago, B.X. 1992, ApJ, 391, L85

Fall, S.M., \& Efstathiou, G. 1980, MNRAS, 193, 189

Forbes, D.A., 2001, these proceedings

Forbes, D.A., Brodie, J.P., \& Grillmair, C.J. 1997, AJ, 113, 1652

Georgakakis, A.E., Forbes, D.A., \& Brodie, J.P. 2001, MNRAS, 324, 785 
Gibson, B., et al. 1999, AJ, 118, 1268

Gnedin, O.Y., \& Ostriker, J.P. 1997, ApJ, 474, 223

Goudfrooij, P., Mack, J., Kissler-Patig, M., Meylan, G., \& Minniti, D. 2001, MNRAS, 322, 643

Harris, G.L.H., \& Harris, W.E. 2000, AJ, 120, 2423

Harris, W.E. 1991, ARAA, 29, 543

Harris, W.E., Harris, G.L.H., \& McLaughlin, D.E. 1998, AJ, 115, 1801

Kissler-Patig, M. 1997, A\&A, 319, 83

Kissler-Patig, M., Brodie, J.P., \& Minniti, D. 2001, A\&A, in preparation

Marleau, F. R., Graham, J. R., Liu, M. C., \& Charlot, S. 2000, AJ, 120, 1779

McLaughlin, D.E. 1999, AJ, 117, 2398

Miller, B.W., Whitmore, B.C., Schweizer, F., \& Fall, S.M. 1997, AJ, 114, 2381

Murali, C., \& Weinberg M. 1997, MNRAS, 288, 767

Ng, Y.K. , \& Bertelli, G. 1998, A\&A, 329, 943

Ostrov, P., Geisler, D., \& Forte, J. C. 1993, 105, 1762

Puzia, T.H., Kissler-Patig, M., Brodie, J.P., \& Huchra, J.P. 1999, AJ, 118, 2734

Puzia, T.H., Zepf, S.E., Kissler-Patig, M., Hilker, M., Minniti, D., \& Goudfrooij, P. 2001, A\&A, in preparation

Rhode, K.L., \& Zepf, S.E. 2001, AJ, 121, 210

Rosenberg, A., Saviane, I., Piotto, G., \& Aparicio, A. 1999, AJ, 118, 2306

Sarazin, C. L., Irwin, J. A., \& Bregman, J.N. 2000, ApJ, 544, 101

Smith, L.J., \& Gallagher, J.S. 2001, MNRAS, in press (astro-ph/0104429)

Spitzer, L. 1987, Dynamical Evolution of Globular Clusters (Princeton: Princeton University Press)

van den Bergh, S. 1990, in Dynamics and Interactions of Galaxies, ed. R Wielen (Berlin: Spinger), 492

Vazdekis, A., Salaris, M., Arimoto, N., \& Rose, J.A. 2001, AJ, 549, 274

Vesperini, E. 1998, MNRAS, 299, 1019

Vesperini, E. 2001, MNRAS, 322, 247

Whitmore, B.C. 1997, The Extragalactic Distance Scale, ed. M. Livio (Cambridge: Cambridge University Press), 254

Whitmore, B.C., Miller, B.W., Schweizer, F., \& Fall, S.M. 1997, AJ, 114, 1797

Whitmore, B.C., et al. 1999, AJ, 118, 1551

Woodworth, S.C., \& Harris, W.E. 2000, AJ, 119, 2699

Worthey, G., Dorman, B., \& Jones, L. A. 1996, AJ, 112, 948

Zepf, S.E. 2001, in The Shapes of Galaxies and Their Halos, in press

Zepf, S.E., \& Ashman, K.M. 1993 MNRAS, 264, 611

Zepf, S.E., Ashman, K.M., \& Geisler, D. 1995, ApJ, 443, 570

Zepf, S.E., Geisler, D., \& Ashman, K.M. 1994, ApJ, 435, L117

Zepf, S.E., et al. 1999, AJ, 118, 752

Zepf, S.E., et al. 2000, AJ, 120, 2928

Zhang, Q., \& Fall, S.M. 1999, ApJ, 527, 81 